\title{
EMPIRICAL STUDY ON CONGESTED SUBWAY TRANSFER TRAFFIC PATTERNS
}

\author{
MENG-CONG ZHENG \\ Department of Industrial Design, National Taipei University of Technology, Taiwan
}

\begin{abstract}
Taipei Main Station of Taipei Mass Rapid Transit is the busiest transport hub in Taiwan in terms of ridership. Its complex layout and high number of passengers frequently lead to congested transfer traffic patterns. This study examined passengers' walking trajectories and behaviours and the relationship between crowding and train movement at the transfer concourse on floor B2 of Taipei Main Station to understand the factors of interference and congestion during traffic flow. An improvement plan was subsequently proposed. This study observed that because more passengers situated themselves in the middle cars than the front and rear cars, most boarding and alighting passengers used specific escalators to enter and exit the platform level. In addition, passengers' walking flow tended to be affected by their personal moving distances, the movement of other passengers and traffic volume. Transfer passengers preferred to use escalators or stairs closer to them, resulting in poor traffic diversion inside the platform. In particular, congestion frequently occurred at the fork near the $\mathrm{T}$ junction, where most passenger interferences were recorded. Passengers tended to lean against walls or walk between pillars to mitigate the conflicting flow of movement among them. Other walking trajectory factors included the locations and directions of escalators, stairs and turnstiles. This study used Unity3D software to construct three traffic diversion proposals based on observation records. The proposals were used to simulate and verify improved traffic patterns and mitigate interference. The simulations revealed that moderate changes in the upward and downward directions of escalators could facilitate smoother transfer traffic patterns. Escalators with traversing directions that better adhere to passengers' traffic patterns may substantially increase passengers' walking speeds regardless of the direction they are coming from, thereby effectively mitigating congestion at the $\mathrm{T}$ junction.

Keywords: non-participant observation, patterns of behaviour, subway transfer, Unity $3 D$.
\end{abstract}

\section{INTRODUCTION}

Taipei Station is an underground station and is the union station for Taiwan Railway, Taiwan High Speed Rail and Taipei Mass Rapid Transit; it is a crucial hub for public transportation in Taipei. On average, 1.95 million people ride the Taipei MRT (mass rapid transit) system every day, and the average number of people entering or leaving Taipei MRT station every day is 0.308 million. Taipei MRT station is the largest MRT station in Taipei; its second underground floor features access to the Bannan Line, Tamsui-Xinyi Line, Exit 8 of Taipei MRT station, an underground market, a food court and a hall. Therefore, a seemingly endless stream of people can be observed there. Dynamic paths for MRT passengers are interlaced with one another. Pedestrian preferences such as speed, distance and location are often influenced by people's personality, current situation and external environment. In addition, pedestrians may be influenced by social forces, e.g. other pedestrians in their proximity; therefore, they may choose an ideal path to reach their destination. Age, gender, mobility, group size, time points during a day and location can significantly influence pedestrian movement [1]. The course of pedestrian behaviour can divided into strategic, tactical and operation stages [2]. In the first stage, pedestrians determine the behaviour that they will present. In the second stage, the pedestrians implement their short-term decisions according to the strategy adopted in the previous stage to determine a location and path where the behaviour will occur; subsequently, the pedestrians return 
to the first stage to plan once more and to undertake two-way communication. In the third stage, the pedestrians make a decision to walk at a certain speed or to wait. On the basis of the interactions between the three stages, why the pedestrians choose a time point or a type of behaviour can be determined. At a congested traffic station, dynamic lines often interfere with one another. The investigation about passenger flow in Taipei MRT station indicated that the transitional hall on the second underground floor is the main transitional space [3]. Therefore, passenger density on the second floor is higher than on other floors. Without an adequate design, passengers' moving paths can be in a state of chaos, thereby reducing the convenience of public transport and influencing passengers' perceptions. The moving path in the transitional hall of the second underground floor is a T-shape intersection at an angle of $90^{\circ}$. A 90 -degree moving path can cause passengers to move chaotically or slowly [4]. Taipei MRT station is Taiwan's busiest station in terms of passenger numbers. On weekdays or holidays, numerous passengers use the services provided by Taipei MRT station. In particular, at rush hours, the moving path in the transitional hall on the second underground floor often becomes chaotic.

In this study, the transitional space on the second underground floor of Taipei MRT station was the research site. This study explored the relationship between the space of the station and passengers' moving paths, and analysed how entrances and exits such as ticket gates, escalators and stairs and environmental objects, e.g. pillars and walls, influence passengers' moving paths. A field survey on passengers' moving paths was conducted. During the survey, passengers' disruptive behaviour was recorded, and the characteristics of passengers' moving paths and the distribution of passenger flow were analysed. Finally, a solution was proposed to improve passengers' moving paths. A software package-Unity3D was used to simulate passenger flow and to assess the effectiveness of various solutions for improving passengers' moving paths.

\section{METHOD}

Taipei MRT station is the most crucial transportation hub in Taipei and provides services to the largest number of passengers in Taiwan; therefore, the results of this study regarding station space and passenger flow are representative. Currently, in the underground transitional space of Taipei MRT station, the passenger flow division facilities are guide lanes, fixed fences and mobile fences (Fig. 1).

The first stage was nonparticipant observation. The experimenters wore minicameras to observe passengers who took the escalator on a platform during weekdays but not during specific holidays or consecutive holidays. The observation period was from 6 to 7 p.m.; these are the peak hours as stated by Taipei MRT station. At each start point, 40 passengers were observed, and a total of 320 passengers were observed. The observation included three parts:

(a) The number of passengers at each start point was recorded to understand the condition of each flow division facility.

(b) The moving trajectory of passengers was recorded from the start point to the end point to understand the characteristics of the transitional moving paths and preferences for start and end points. The moving path of passengers was recorded using spatial coordinates to identify areas with the most interlaced moving paths in the transitional hall.

(c) All disruptive behavior of transitional passengers was recorded to understand its cause and the responses of other passengers to the disruptive behavior. 


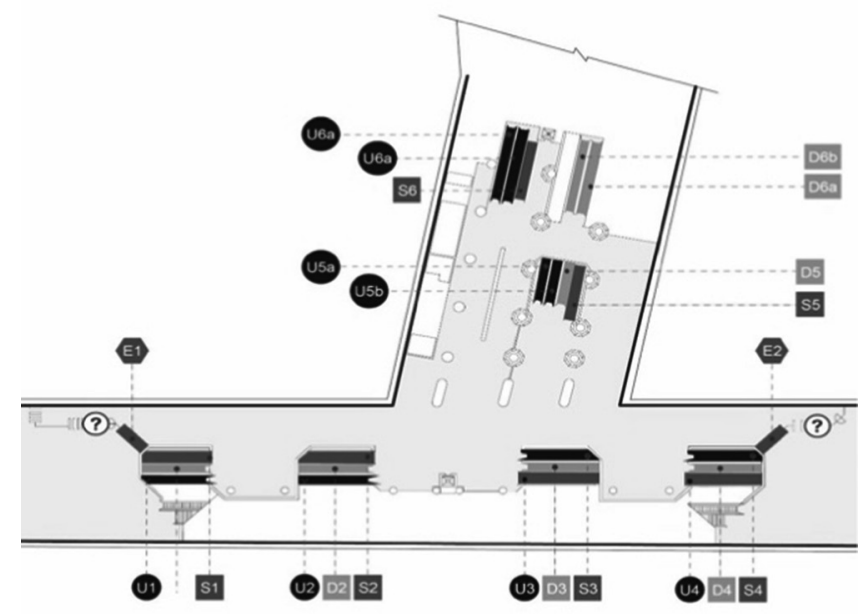

Figure 1: Start and end points in the research site.

The second stage was a flow division simulation experiment. The tool used for the simulation experiment was Unity3D, which is a three-dimensional electronic game containing visualized buildings and 3D interactive animations (Fig. 2). Nav Mesh and Nav Mesh Agent functions were used to help passengers read the Nav Mesh Agent and operate in Nav Mesh. In addition, an endpoint design was adopted to identify the shortest path to reach a destination. First, according to the observation results including the number of passengers at each start and end point, the start and end points chosen by passengers, the characteristics of passengers' transitional moving paths and passengers' transitional behaviour, a simulation model was constructed. Finally, according to the results of the simulation experiment, a solution for dividing passenger flow and improving moving paths on the second underground floor in Taipei MRT station was proposed.

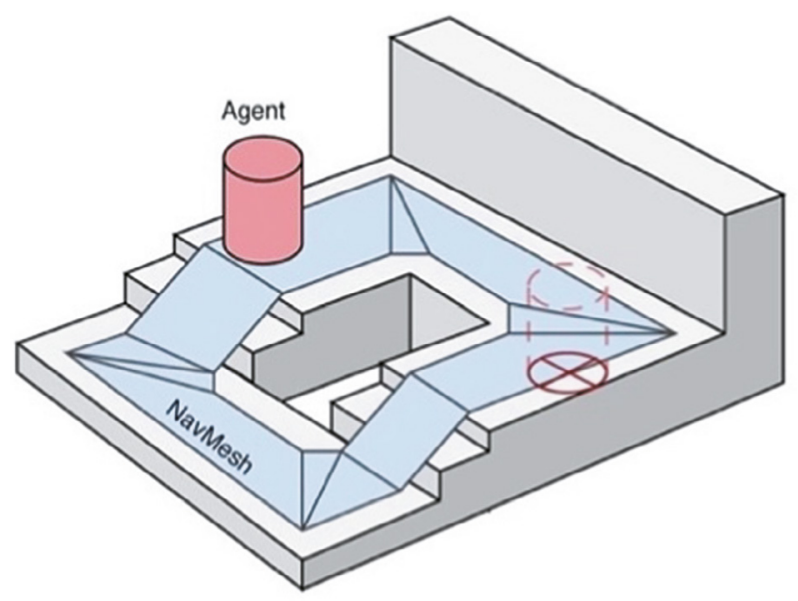

Figure 2: Unity3D. 


\section{RESULTS AND DISCUSSION}

To elucidate the observed passenger flow at each start point, the escalator as Code $U$ and stairs as Code $\mathrm{S}$ in the same region were called US. The results showed that 30,000 passengers entered into the transitional space on the second underground floor from the ticket gates and the start points of the Bannan Line and Tamsui-Xinyi Line. The start point with the largest passenger flow was US6, followed by US5, US3, US2, E1, E2, US1 and US4. In addition, $39 \%$ of the passengers departed from the Bannan Line, $37 \%$ of the passengers departed from the Tamsui-Xinyi Line and $24 \%$ of the passengers entered into Taipei MRT station from the outside (Fig. 3).

At this stage, the amount of time taken by passengers from each start point to the transitional space on the second underground floor was investigated, as well as the intervals for a crowding cycle. As shown in Fig. 4, for passengers who departed from the escalator for the Bannan Line, the start point with the longest crowding period was ${ }_{3}$, followed by ${ }_{2}$, (U1) and (44. The ranking of stairs was identical to the ranking of escalators. For passengers who departed from the escalator for the Tamsui-Xinyi Line, the start points with the longest

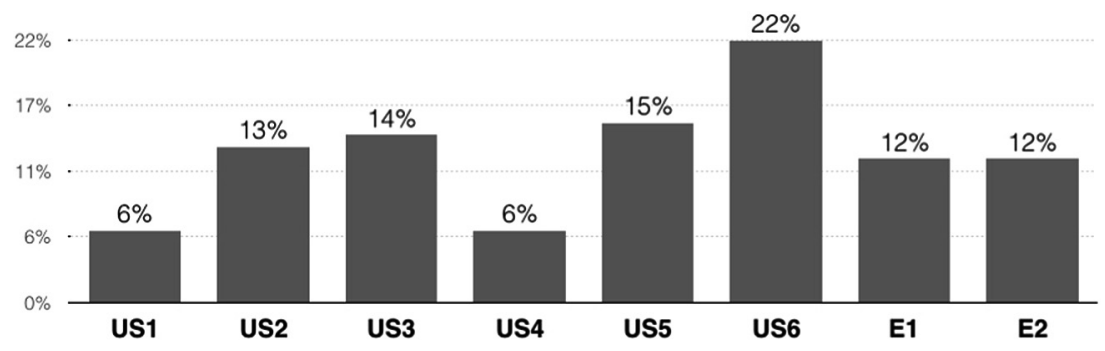

Figure 3: Percentage of passengers at each start point.

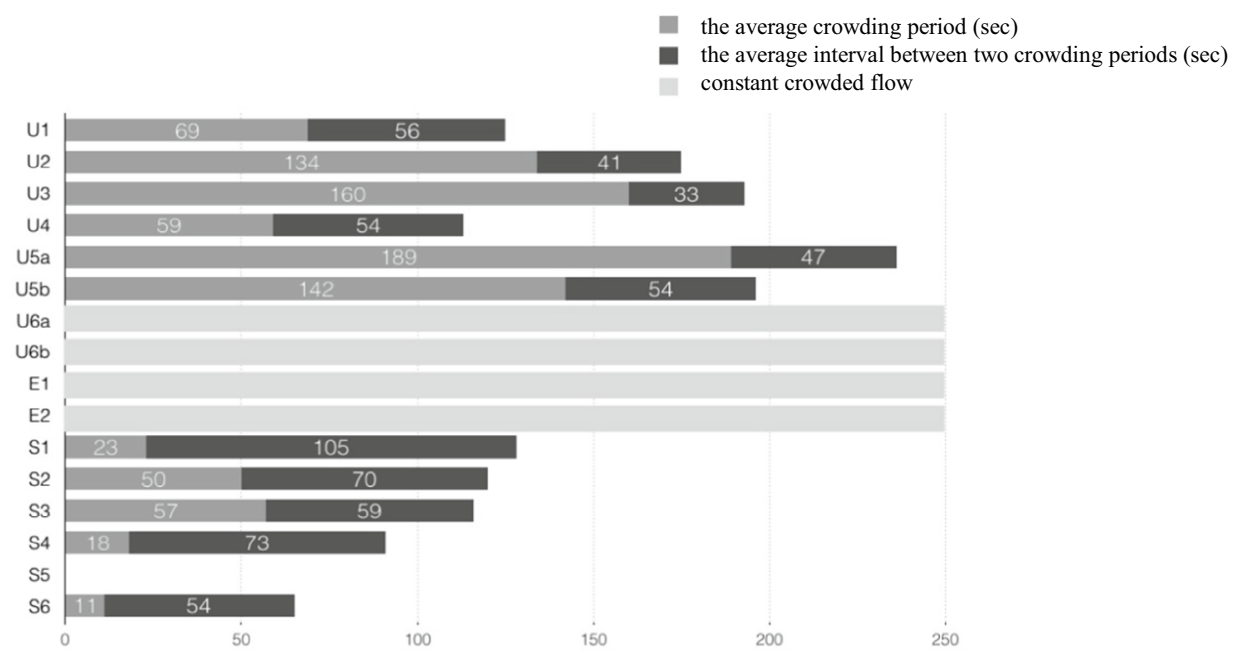

Figure 4: Crowding periods for each start point and the intervals between two crowding periods. 
continuous crowding period were 163 and 066 , followed by 150 and 159 . The ranking of stairs was identical to the ranking of escalators. At $\mathbf{E} 1$ and E2, crowded flow constantly occurred. At 66 , U6, E1 and E2, the start points were connected to ticket gates and the outside; therefore, large crowds were constantly observed. The results indicated that for a larger passenger flow, a single crowding period was longer and the interval between two crowding periods for different MRT rides was shorter. In addition, the manner in which passengers stood on an escalator influenced the crowding period. If more passengers stood on one side of the escalator, the crowding period for one MRT ride was longer.

To understand the characteristics of transitional moving paths and the interlacing of moving paths, the moving paths from the Bannan Line, Tamsui-Xinyi Line and ticket gates were overlapped. The blue line represents the moving path to the Bannan Line, the red line represents the moving path to the Tamsui-Xinyi Line and the green line represents the moving path to the ticket gates.

As shown in Fig. 5, among passengers from Regions $\mathrm{B}$ to $\mathrm{K}$ along the $\mathrm{X}$ axis, the passengers who moved toward the Bannan Line and Tamsui-Xinyi Line tended to move toward Code 3 along the $\mathrm{Y}$ axis; and the passengers who moved toward the ticket gates tended to move toward Code 4 along the $\mathrm{Y}$ axis. From Regions $\mathrm{L}$ to $\mathrm{Q}$ along the $\mathrm{X}$ axis, passengers' moving trajectories appeared to be radial. Therefore, Regions $\mathrm{L}$ to $\mathrm{Q}$ were the intersections of the moving paths, where the moving paths in various directions often conflicted with one another and had dispersed and complex trajectories. Among passengers in Regions $\mathrm{R}$ to $\mathrm{V}$ along the $\mathrm{X}$ axis, the passengers who moved toward the Tamsui-Xinyi Line tended to approach Code 4 along the $\mathrm{Y}$ axis and the passengers who moved toward ticket gates tended to approach Code 3 along the $\mathrm{Y}$ axis.

The moving paths near Exit 22 which from coordinates $(\mathrm{W}, 4)$ to coordinates $(\mathrm{V}, 1)$ were chaotic by passengers start from (04. In addition, the paths near the escalator were interlacing, as were the paths near coordinates $(\mathrm{O}, 4)$. Passengers who departed from the Tamsui-Xinyi Line and transferred to the Bannan Line typically concentrated on 02 and (03. Therefore, a large crowd gathered near coordinates $(\mathrm{L}, 4)$ and coordinates $(\mathrm{O}, 1)$. Passengers who departed from 05 were easily influenced by passenger flow from coordinates $(\mathrm{P} 1,5)$ to coordinates

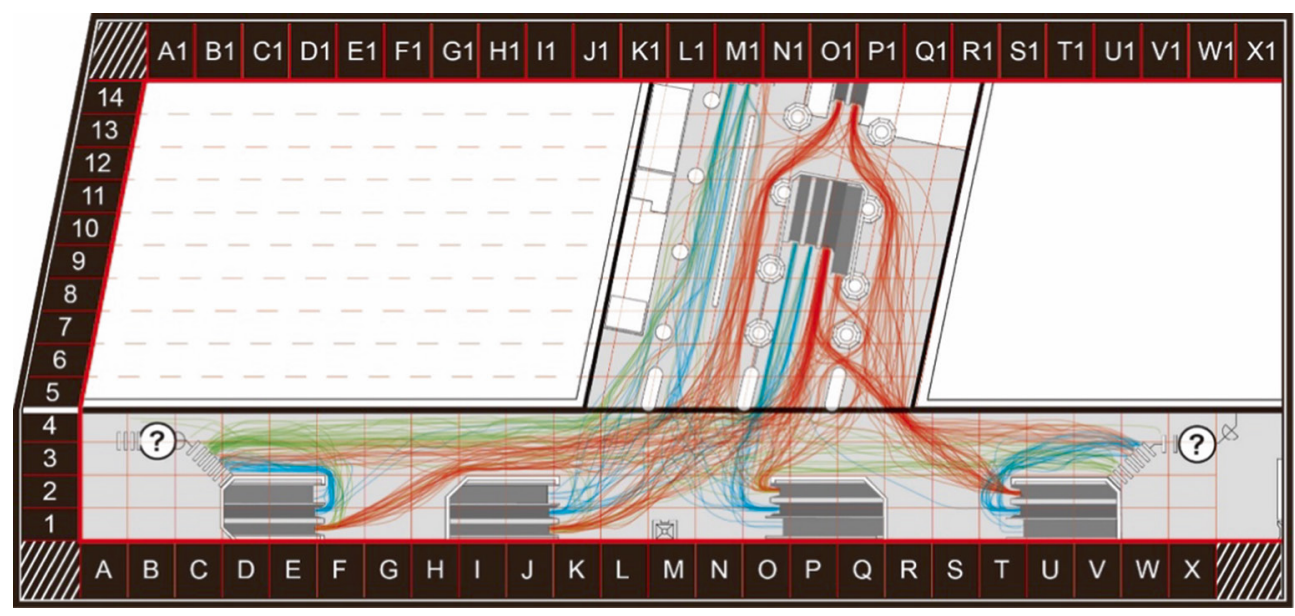

Figure 5: Diagram for passengers' overlapped moving paths. 
(P1, 9); accordingly, passengers who transferred to the Bannan Line often chose destinations in coordinates $(\mathrm{K}, 1)$ and coordinates $(\mathrm{O}, 1)$. Transitional moving paths often conflicted with one another, particularly at entrance and exit gates, escalators and stairs.

According to Fig. 6 regarding behavioural types and the number of behavioural types at various start points, during the transitional processes, disruptive behaviour occurred 545 times. Specifically, the most disruptive behaviour was path change total 311 times, followed by slowing down total 140 times, dodging, stopping moving and collisions. Therefore, when passengers faced conflicts and disruption, they often changed their moving paths to avoid physical collision. When the Bannan Line served as the start point, the most disruptive behaviours occurred at (U2, followed by 11 and U3. When the Tamsui-Xinyi Line served as the start point, the most disruptive behaviours occurred at 150 , followed by 559 , 66 and 165 . When the entrance gate served as the start point, the most disruptive behaviours occurred at $\mathbf{E}$, followed by E2.

By overlapping disruptive behaviour, a region of darker colour indicated that disruptive behaviour occurred more frequently. As shown in Fig. 7, at a turn or intersection, passengers headed in various directions and were influenced by various directions of moving flow, thereby presenting more disruptive behaviour. Disruptive behaviour occurred frequently between coordinates $(\mathrm{L}, 4)$ and coordinates $(\mathrm{O}, 3)$. Particularly, in the region between coordinates $(\mathrm{N}, 4)$ and coordinates $(\mathrm{O}, \mathrm{N})$, disruptive behaviour occurred most frequently. Outside the region between coordinates $(\mathrm{L}, 4)$ and coordinates $(\mathrm{O}, 3)$, the moving paths were almost straight and bidirectional where disruptive behaviour occurred infrequently. Passengers often encountered disruptive behaviour at the turning corners on the top of the first escalator at coordinates $(\mathrm{E}, 3),(\mathrm{J}, 3),(\mathrm{T}, 3)$ and $(\mathrm{O}, 3)$. Therefore, moving paths were often disrupted at turning corners.

In Unity3D flow division simulation experiment, the space of the station was divided into 11 regions (Fig. 8). By comparing the average rates in various regions before and after the experiment, the effectiveness of each solution for improving moving paths was determined.

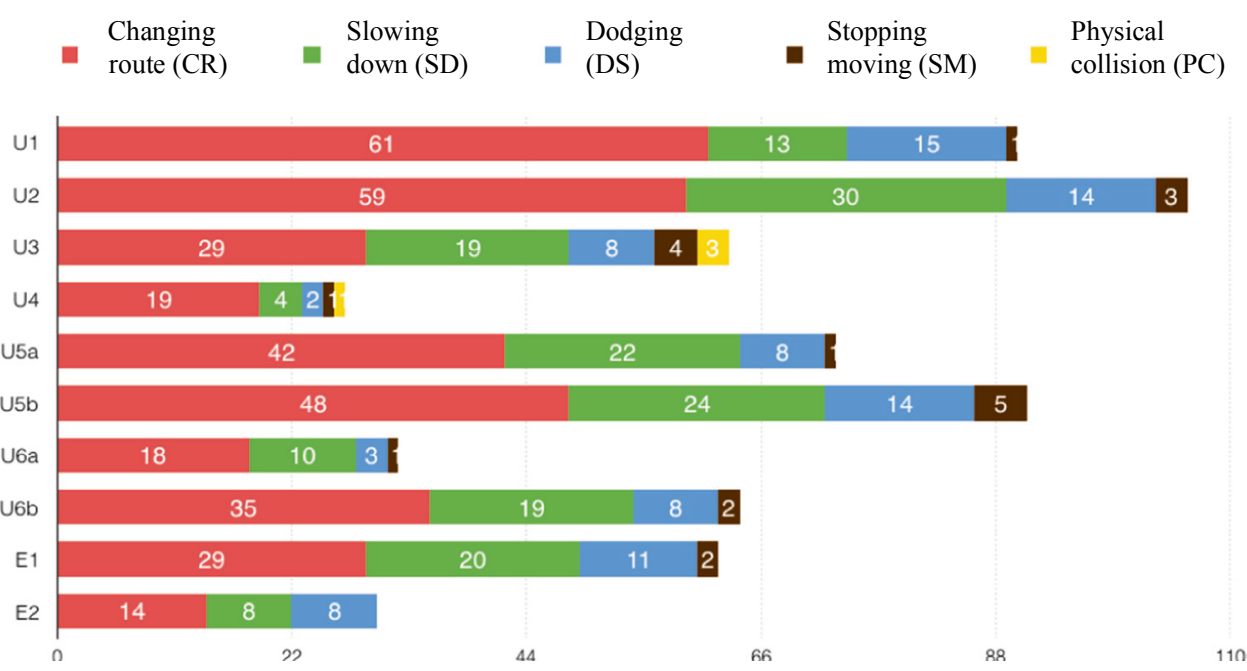

Figure 6: Behavioural types and the number of behavioural types at various start points. 


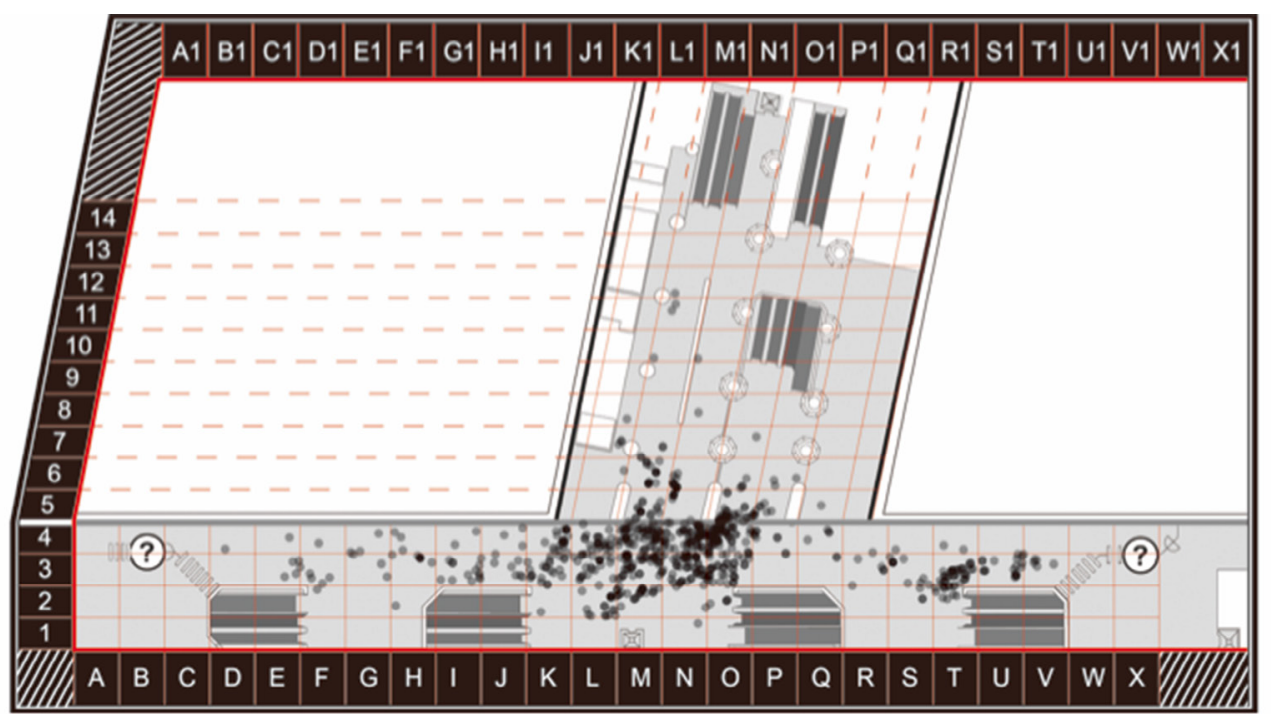

Figure 7: Diagram for disruptive behaviour.

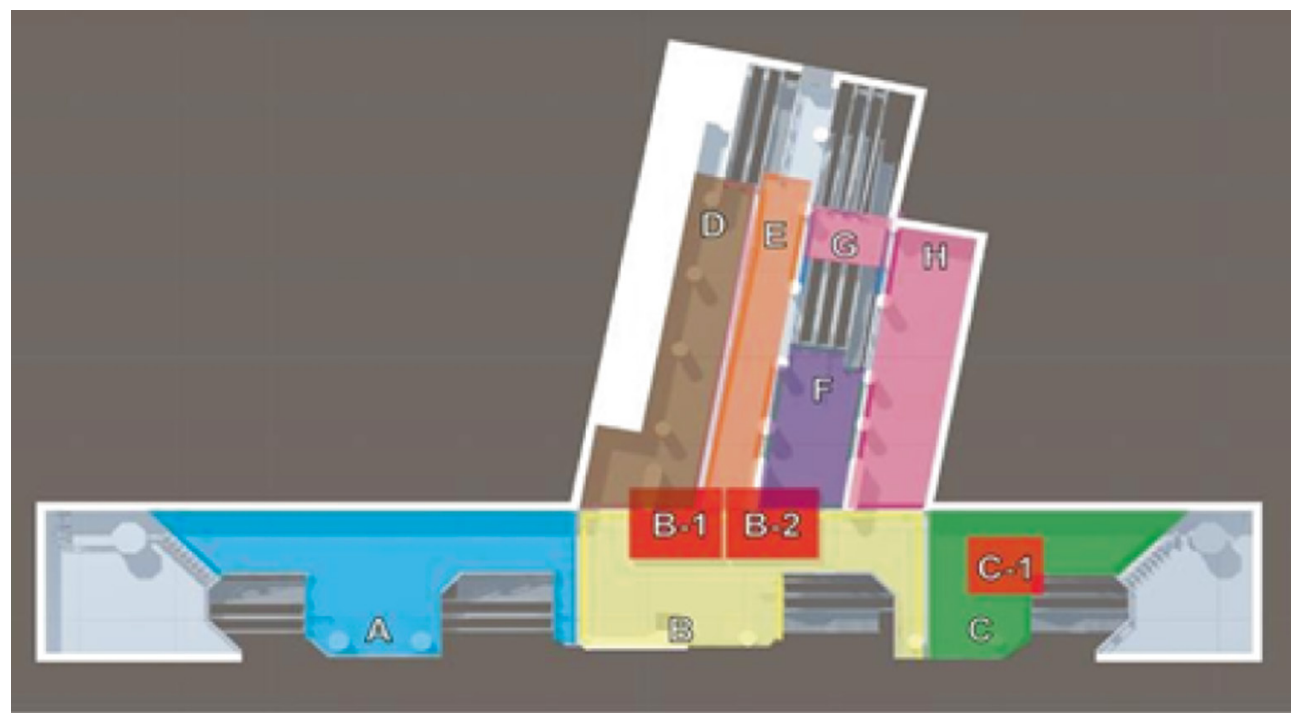

Figure 8: Diagram for various regions.

For the control group (Fig. 9), the first three regions with the slowest walking speed were Regions B-2, F and B. Region B-2 was a turning corner. There, passengers were influenced by interlaced moving paths and visual angles; therefore, this was where disruptive behaviour most frequently occurred. Regions B-1 and B-2 formed Region B where numerous moving paths intersected and numerous T-shape intersections existed. In this region, the average walking speed was low. In Region F, the escalator was located at the centre of the station; most passengers chose Escalator D5 as their end point; therefore, a queue often formed to slow the average walking speed in this region. Three simulation solutions were proposed: 


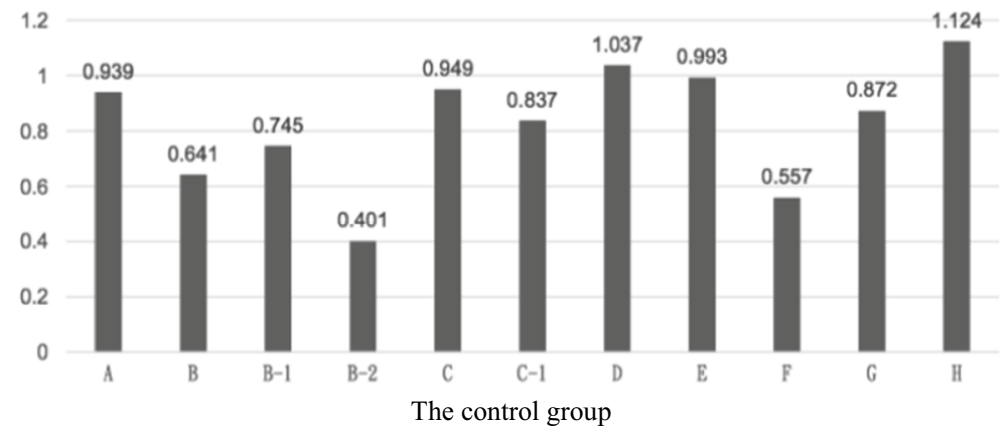

Figure 9: Walking speed at each region.

Solution A: Change the moving paths where disruptive behaviour often occurs. In Stage 1 , this study discovered that frequent disruptive behaviour occurred in the regions near turning corners or intersections. When the Bannan Line served as a start point, passengers who departed from ${ }^{4}$ did not pass a congested T-shape intersection. However, passengers who transferred to the Bannan Line preferred to choose $U_{2}$ and $\left(\mathbf{U}_{3}\right.$ as their start points; therefore, the passengers typically passed a turning corner or intersection. When the Tamsui-Xinyi Line served as a start point, passengers who departed from 16 and 66 and headed toward E1 typically chose to move through pillars and avoid interlaced moving paths. However, passengers who departed from 66 and 160 and headed toward the Bannan Line preferred to choose $\left(12\right.$ and $\left(U_{3}\right.$ as the end points instead of 11 (Fig. 10). Solution A can be used to

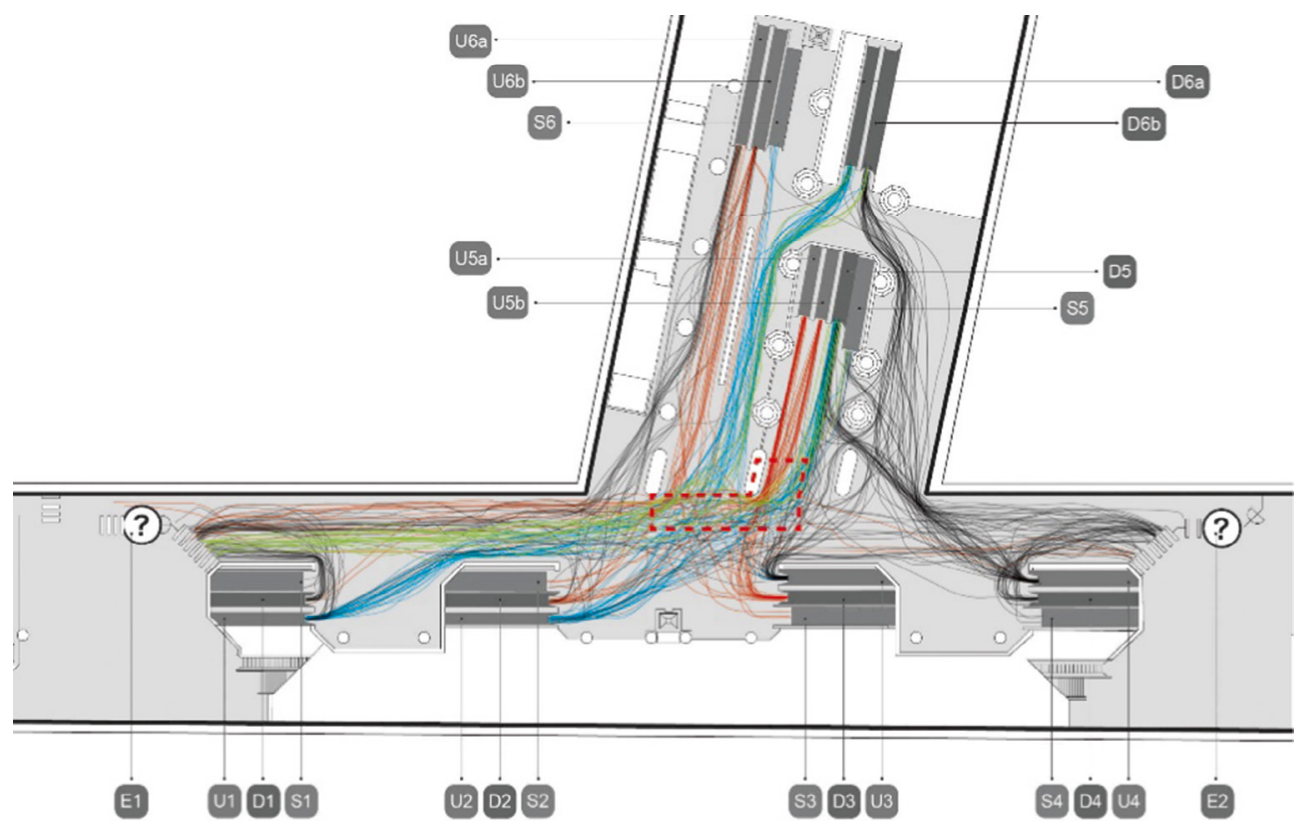

Figure 10: Where disruptive behaviour occurred. 
maintain passenger flow at various start points and to adjust moving paths according to the end points chosen by passengers, thereby reducing congestion and the number of interlaced moving paths at T-shape intersections. The flow division adjustment is as follows: For passengers who departed from the Bannan Line $U_{2}$ and headed toward the Tamsui-Xinyi Line at 05 and (06, passengers at 42 were diverted to 04 . For passengers who departed from the Tamsui-Xinyi Line 66 and 66 and headed toward the Bannan Line at (22 and (03, passengers at 03 were diverted to (1) and D2.

Solution B: Change passenger flow at the start points. Table 1 presents passenger flow at start points of Bannan Line, Tamsui-Xinyi Line and the entrance gate. Passengers who took Bannan Line typically chose US2 and US3 as their start points. The two start points were close to the region where numerous interlaced moving paths existed. Start points chosen by passengers were directly related to the paths they transferred to. For various start points, inadequate flow division can cause moving paths to concentrate on a specific region, resulting in interlaced moving paths and congestion. Therefore, at this stage, passenger flow at various start points was adjusted on the basis of Solution A to understand the influence of the change of passenger flow at the start points on transitional moving paths and to explore the effectiveness of Solution A for improving moving paths. Because passenger flow was similar at various start points for the Tamsui-Xinyi Line, at this stage, the passenger flow at each start point for the Bannan Line was adjusted to $8.45 \%$; passenger flow at other start points was not adjusted.

Solution C: Change the operating direction of an escalator. According to the results obtained in Stage 1 of this study, the spaces, layout and operation of facilities were directly related to the smoothness of passengers' transitional moving paths. As shown in Fig. 11, the portions marked by red circles were influenced by the layout of facilities, resulting in overlapped and interlaced moving paths. The operating directions of escalators also influenced moving paths (Table 2). In this simulation experiment, the operating directions of escalators were changed to reduce the occurrence of interlaced moving paths at turning corners and to explore the effectiveness of the solution for improving moving paths.

According to the results of the simulation experiment regarding average speeds in various regions (Fig. 12), Solution C presented the optimal performance; among 11 regions, for the average speeds in eight regions, the performance of Solution $\mathrm{C}$ was superior to the

Table 1: Passenger flow at various start points.

\begin{tabular}{lccc}
\hline Before adjustment & \multicolumn{3}{c}{ After adjustment } \\
\hline Code of escalator & Percentage & Code of escalator & Operating direction (\%) \\
\hline US1 & 6.28 & US1 & 8.45 \\
US2 & 10.67 & US2 & 8.45 \\
US3 & 10.75 & US3 & 8.45 \\
US4 & 6.12 & US4 & 8.45 \\
U5a & 11 & U5a & 11 \\
U5b & 9.2 & U5b & 9.2 \\
U6a & 9 & U6a & 9 \\
U6b & 10.8 & U6b & 10.8 \\
E1 & 12.5 & E1 & 12.5 \\
E2 & 13.2 & E2 & 13.2 \\
\hline
\end{tabular}




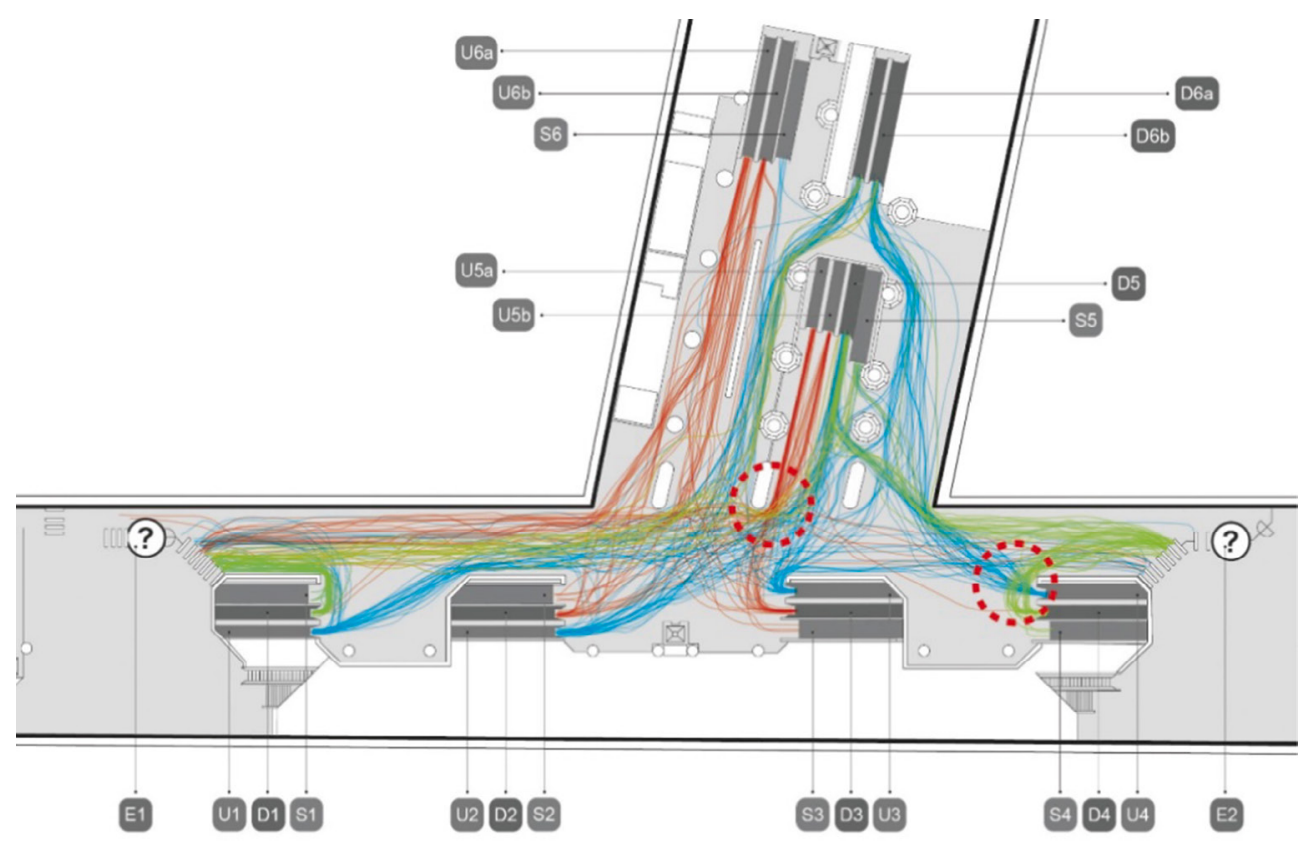

Figure 11: Where facilities overlapped and interlaced moving paths.

Table 2: Code of escalator and operating direction.

\begin{tabular}{lccc}
\hline Before adjustment & \multicolumn{3}{c}{ After adjustment } \\
\hline Code of escalator & Operating direction & Code of escalator & Operating direction \\
\hline U4 & $\mathrm{Up}$ & D4 & Down \\
D4 & Down & U4 & Up \\
U5a & $\mathrm{Up}$ & D5 & Down \\
D5 & Down & U5a & Up \\
U5b & $\mathrm{Up}$ & U5b & $\mathrm{Up}$ \\
\hline
\end{tabular}

performances of the control group and other flow division solutions. Solution B presented the second most optimal performance; for the average speeds in five regions, the performance of Solution B was superior to the performance of the control group. Solution A presented the least optimal performance; for the average speeds in four regions, the performance of Solution A was superior to that of the control group. Therefore, inadequate flow division can result in disruptive moving paths. This study explored the amount of time required to move from a start point to an end point. A small amount of time indicated a low degree of disruption in moving paths. For Solution $\mathrm{C}$, the average amount of time required to move from $(41$ or $(42$ to an end point was the shortest. Considering moving from four start points at $(11)$ and 


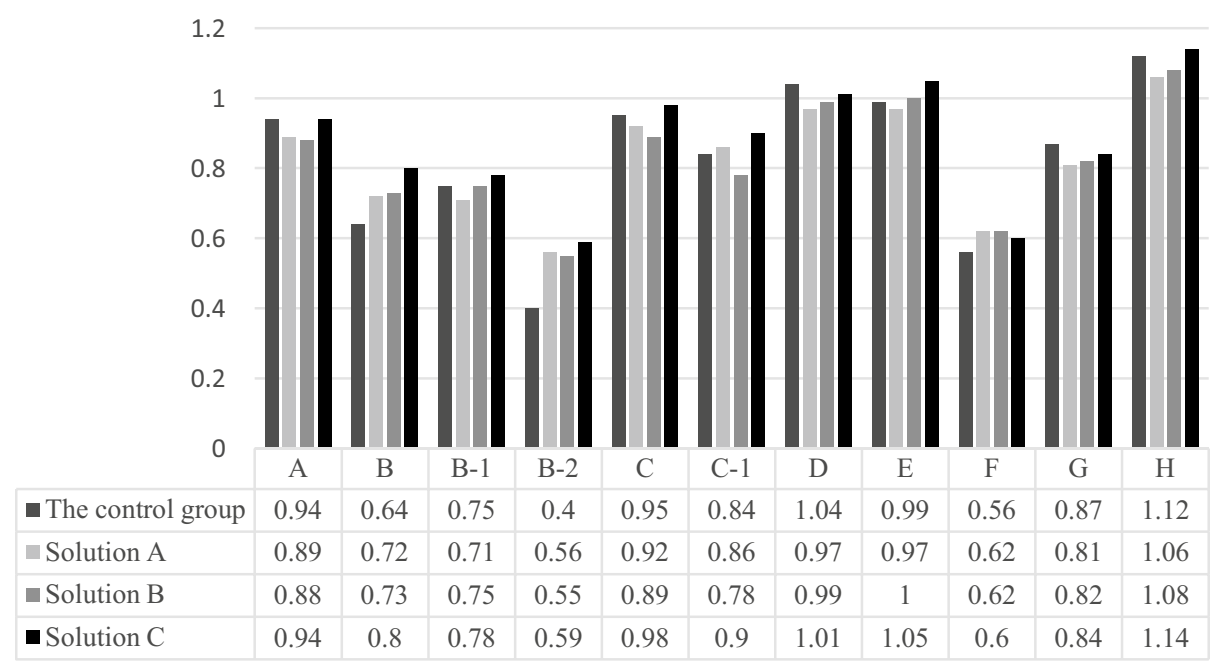

Figure 12: Comparison between the experimental group and the control group regarding three solutions.

six start points at $(22$ to an end point, Solution $\mathrm{C}$ was superior to other solutions. In Solution $\mathrm{C}$, the amount of time required for moving from a start point to (05, E2 or S5 significantly reduced. Therefore, adjusting the operating directions of escalators at U5a, U5b and (05 reduced disruption in the moving path from (1) to D5. In addition, the congestion in the surrounding areas reduced, and the moving paths heading toward $\mathbf{E} 2$ improved. Considering the average amount of time required to move from 43 to an end point, the control group presented the optimal performance. For four end points, the control group required a smaller amount of time than the experiment group. Considering the average amount of time required to move from $15 \mathrm{a}$ and $15 \mathrm{~s}$ to an end point, Solution $\mathrm{C}$ presented the optimal performance. For the average amount of time required to move from four start points at $55 \mathrm{a}$ and three start points at 55 to an end point, Solution $\mathrm{C}$ was superior to other solutions. Considering the average amount of time required to move from $15 \mathrm{a}$ and 150 to an end point, the three solutions in the experiment group were superior to the control group. Therefore, the three solutions effectively reduced the amount of time required to move toward the Bannan Line. (55 Considering the average amount of time in seconds required to move from 16 to an end point, the control group and Solution C presented similar performances; however, Solution A required the highest amount of time. With Solutions A and B, the adjustment of flow division increased the number of passengers who arrived at (01) and (22 and aggravated the disruption in moving paths toward (D2, S2 and E1, thereby slowing passengers' walking speed. Considering the average amount of time in seconds required to move from 66 and $\mathbf{E} 1$ to an end point, Solution $\mathrm{C}$ presented the optimal performance. For the average amount of time required to move from five start points at 16 and five start points at $\mathbf{E}$ to an end point, Solution $\mathrm{C}$ was superior to other solutions. Adjusting the operating directions of escalators resulted in smoothness of the moving path toward (05 and 55. The average amount of time required to move from (44) to an end point did not significantly differ from that required to move from $\mathbf{E}_{2}$ to an end point. However, Solution C required more time to arrive at 05 and $\mathbf{E 2}$ than other solutions. 
Therefore, adjusting the operating directions of escalators caused disruption in moving paths from $\left(14\right.$ and $E_{2}$ to D5; therefore, Solution $C$ required more time to move from $\left(44\right.$ and $E_{2}$ to D5 than other solutions.

\section{CONCLUSION}

Through observing passenger flow at various start points, results of this study indicated that the interval between two MRT rides, passenger flow, space size and the escalator location influenced whether moving paths were interlaced with one another or congested with people. This study discovered that passenger flow at each start point varied. Numerous people chose start points at transitional intersections; accordingly, the space was overloaded with people, resulting in congestion. For example, among passengers at various start points near the Bannan Line, numerous passengers chose start points close to transitional intersections. However, the choice of a start point was related to the number of passengers in an MRT car. The number of passengers in a middle MRT car was higher than that in the first or last MRT car. Therefore, passengers were typically concentrated at escalators near middle MRT cars. In addition, passengers standing on one side of an escalator easily prolonged the crowding period; accordingly, a large crowd formed after successive MRT trains arrived. When passengers' moving paths were disrupted, the passengers changed their moving paths or routes to dodge other passengers. The passengers often changed to a direction opposite to the disruption or a direction toward an area with a low passenger density. Slowing down of walking speed often accompanied path change. Passengers amid numerous people often slowed their walking speed to identify their future walking directions or to yield. In addition, passengers typically walked on the right-hand side; accordingly, two opposite passenger flows formed. A passenger flow was divided into two flows near turning corners or intersections. Passengers often walked along pillars or walls to avoid being disrupted by other passengers. When the station was crowded, most passengers chose a shortcut to reach their goal and did not detour around the crowd. Therefore, congestion easily occurred because of inadequate flow division. In the simulation experiment, the three flow division solutions reduced the average time required to move from U5a and U5b to end points. Therefore, these solutions reduced disruption in moving paths along T-shape intersections. Among the three flow division solutions, only Solution C substantially reduced disruption in moving paths near turning corners or intersections and substantially improved the moving speed near T-shape intersections.

With Solution C, the operating directions of escalators and passengers' moving paths were adjusted. Therefore, the operating directions of escalators were closely related to passengers' transitional moving paths. In addition, an excellent layout of facilities improved the coherence of passengers' transitional moving paths. Solution $\mathrm{C}$ required more time to move from E2 to D5 than other solutions; therefore, Solution C caused more disruptions than other solutions. If passengers who departed from E2 chose D6a and D6b as their end points, the disruption reduced. Therefore, a flow division solution cannot improve a single moving path. However, Solution C effectively reduced disruption in moving paths in the station. The results of the simulation experiment indicated that changing passenger flows at various start points improved moving paths to a limited degree. To improve problems regarding flow division, the relationship between facilities, passengers' moving paths and passenger flow must be considered. The investigation method adopted in this study can serve as a reference for the management and design of Taipei Station. 


\section{REFERENCES}

[1] Alexandra, W., Human movement behaviour in urban spaces: implications for the design and modelling of effective pedestrian environments. Environment and Planning B: Planning and Design, 31, pp. 805-828, 2004. DOI: 10.1068/b3060

[2] Hoogendoorn, S.P., Bovy, P.H. \& Daamen, W., Walking infrastructure design assessment by continuous space dynamic assignment modeling. Journal of Advanced Transportation, 38(1), pp. 69-92, 2004. DOI:10.1002/atr.5670380106

[3] Wen, K.-C., A study of passenger's moving conflict to ameliorate flow by simulationbased evaluation in MRT Taipei Main Station. Master thesis, Chinese Culture University, 2011.

[4] Fan, C.-H., A study on passengers weaving behavior model. Master thesis, TamKang University, 2011. 